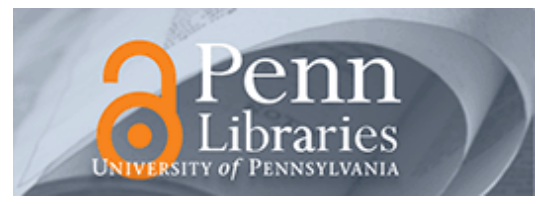

University of Pennsylvania

ScholarlyCommons

Marketing Papers

Wharton Faculty Research

$9-2013$

\title{
Temporal Changes of Neocortical High-Frequency Oscillations in Epilepsy
}

\author{
Allison Pearce \\ University of Pennsylvania \\ Drausin F Wulsin \\ University of Pennsylvania \\ Justin A Blanco \\ Abba M. Krieger \\ University of Pennsylvania \\ Brian Litt \\ University of Pennsylvania
}

See next page for additional authors

Follow this and additional works at: https://repository.upenn.edu/marketing_papers

Part of the Marketing Commons, Medical Neurobiology Commons, Medical Physiology Commons, Neurology Commons, Neurosciences Commons, and the Psychology Commons

\section{Recommended Citation}

Pearce, A., Wulsin, D., Blanco, J., Krieger, A. M., Litt, B., \& Stacey, W. C. (2013). Temporal Changes of Neocortical High-Frequency Oscillations in Epilepsy. Journal of Neurophysiology, 110 (5), 1167-1179. http://dx.doi.org/10.1152/jn.01009.2012

This paper is posted at ScholarlyCommons. https://repository.upenn.edu/marketing_papers/344

For more information, please contact repository@pobox.upenn.edu. 


\title{
Temporal Changes of Neocortical High-Frequency Oscillations in Epilepsy
}

\begin{abstract}
High-frequency $(100-500 \mathrm{~Hz})$ oscillations (HFOs) recorded from intracranial electrodes are a potential biomarker for epileptogenic brain. HFOs are commonly categorized as ripples $(100-250 \mathrm{~Hz})$ or fast ripples $(250-500 \mathrm{~Hz})$, and a third class of mixed frequency events has also been identified. We hypothesize that temporal changes in HFOs may identify periods of increased the likelihood of seizure onset. HFOs $(86,151)$ from five patients with neocortical epilepsy implanted with hybrid (micro + macro) intracranial electrodes were detected using a previously validated automated algorithm run over all channels of each patient's entire recording. HFOs were characterized by extracting quantitative morphologic features and divided into four time epochs (interictal, preictal, ictal, and postictal) and three HFO clusters (ripples, fast ripples, and mixed events). We used supervised classification and nonparametric statistical tests to explore quantitative changes in HFO features before, during, and after seizures. We also analyzed temporal changes in the rates and proportions of events from each HFO cluster during these periods. We observed patient-specific changes in HFO morphology linked to fluctuation in the relative rates of ripples, fast ripples, and mixed frequency events. These changes in relative rate occurred in pre- and postictal periods up to thirty min before and after seizures. We also found evidence that the distribution of HFOs during these different time periods varied greatly between individual patients. These results suggest that temporal analysis of HFO features has potential for designing custom seizure prediction algorithms and for exploring the relationship between HFOs and seizure generation.
\end{abstract}

Keywords

epilepsy, HFO, oscillation, machine learning, classifier

Disciplines

Business | Marketing | Medical Neurobiology | Medical Physiology | Neurology | Neurosciences | Psychology

Author(s)

Allison Pearce, Drausin F Wulsin, Justin A Blanco, Abba M. Krieger, Brian Litt, and William C. Stacey

This technical report is available at ScholarlyCommons: https://repository.upenn.edu/marketing_papers/344 
Articles in PresS. J Neurophysiol (June 12, 2013). doi:10.1152/jn.01009.2012

Temporal Changes of Neocortical High Frequency Oscillations in Epilepsy

Allison Pearce

Drausin Wulsin

Justin A. Blanco

Abba Krieger

Brian Litt

William C. Stacey * $(6,7)$

Running head: Temporal Changes of HFOs in Epilepsy

1. Department of Computer Science, University of Pennsylvania, Philadelphia, PA

2. Department of Bioengineering, University of Pennsylvania, Philadelphia, PA

3. Electrical and Computer Engineering Department, US Naval Academy, Annapolis, MD

4. Department of Statistics, Wharton School of Business, University of Pennsylvania, Philadelphia, PA

5. Penn Epilepsy Center, University of Pennsylvania, Philadelphia, PA

6. Department of Neurology, University of Michigan, Ann Arbor, MI

7. Department of Biomedical Engineering, University of Michigan, Ann Arbor, MI

* - Address correspondence to: William Stacey MD PhD, University of Michigan, Department of

29 Neurology, 1500 E. Medical Center Drive, SPC 5036, Ann Arbor, MI 48109-5036,

30 William.stacey@umich.edu

31

33 Figures: 6

34 Tables: 4 


\section{Abstract}

High frequency $(100-500 \mathrm{~Hz})$ oscillations (HFOs) recorded from intracranial electrodes are a potential biomarker for epileptogenic brain. HFOs are commonly categorized as ripples $(100-250 \mathrm{~Hz})$ or fast ripples $(250-500 \mathrm{~Hz})$, and a third class of mixed frequency events has also been identified. We hypothesize that temporal changes in HFOs may identify periods of

41 increased likelihood of seizure onset. 86,151 HFOs from five patients with neocortical epilepsy

42 implanted with hybrid (micro + macro) intracranial electrodes were detected using a previously

43 validated automated algorithm run over all channels of each patient's entire recording. HFOs

44 were characterized by extracting quantitative morphologic features and divided into four time

45 epochs (interictal, preictal, ictal, and postictal) and three HFO clusters (ripples, fast ripples, and 46 mixed events). We used supervised classification and nonparametric statistical tests to explore

47 quantitative changes in HFO features before, during, and after seizures. We also analyzed

48 temporal changes in the rates and proportions of events from each HFO cluster during these

49 periods. We observed patient-specific changes in HFO morphology linked to fluctuation in the

50 relative rates of ripples, fast ripples, and mixed frequency events. These changes in relative rate

51 occurred in pre- and postictal periods up to thirty minutes before and after seizures. We also

52 found evidence that the distribution of HFOs during these different time periods varied greatly

53 between individual patients. These results suggest that temporal analysis of HFO features has

54 potential for designing custom seizure prediction algorithms and for exploring the relationship 55 between HFOs and seizure generation.

Key Words: Epilepsy, HFO, oscillation, machine learning, classifier 


\section{Introduction}

High frequency oscillations (HFOs) have received increasing interest as a promising

63 biomarker for epileptogenic tissue. HFOs are discrete electrophysiological events that stand out

64 from background activity and are seen in normal as well as epileptic tissue (Engel et al. 2009).

65 They occur in the 100-500 Hz frequency range and generally last on the order of tens of

66 milliseconds. HFOs have conventionally been separated into two classes by frequency: ripples

$67(100-250 \mathrm{~Hz})$ and fast ripples $(250-500 \mathrm{~Hz})$, though a third class of mixed frequency events has

68 been automatically identified in human neocortical epilepsy (Blanco et al. 2010). Ripples were

69 first identified during normal brain functions such as memory consolidation (Buzsaki 1998;

70 Grenier et al. 2001; Siapas and Wilson 1998) but were later identified in epileptic tissue as well

71 (Bragin et al. 2002a).

The study of HFOs may help elucidate mechanisms of seizure generation (Demont-

73 Guignard et al. 2012; Jefferys et al. 2012). Many investigations have focused on the mechanisms

74 generating ripples (Bragin et al. 2004; Bragin et al. 2007; Ylinen et al. 1995) and fast ripples

75 (Dzhala and Staley 2004; Foffani et al. 2007; Ibarz et al. 2010; Menendez de la Prida and

76 Trevelyan 2011). Fast ripples have been more closely linked to pathological activity and localize

77 to the seizure onset zone (Bragin et al. 2002b; Urrestarazu et al. 2007). However, investigations

78 of human intracranial recordings indicate that HFOs in both frequency ranges increase in

79 epileptogenic brain regions (Jacobs et al. 2010; Worrell et al. 2008). Like seizures, HFOs

80 increase after medication reduction, indicating a close link between the two phenomena

81 (Zijlmans et al. 2009). These studies investigated HFOs primarily as a spatial biomarker, as they

82 appear to have great potential for delineating epileptogenic brain. Removal of HFO-generating

83 tissue has been shown to correlate with better outcomes of resective surgery (Akiyama et al. 
84 2011; Jacobs et al. 2010). However, one of the primary challenges in HFO research remains how

85 to distinguish between normal and abnormal HFOs (Engel et al. 2009; Traub 2003); merely

86 identifying fast ripples and ripples is not specific enough, as all types of HFOs are present even

87 in normal human brain tissue (Blanco et al. 2011).

One method of characterizing HFOs that has received relatively little attention is a

90 detailed analysis of their temporal properties in the periods during and surrounding seizures. The

91 seminal research on this subject has been encouraging. An in-vitro study demonstrated increased

92 ripple and fast ripple activity before seizures (Khosravani et al. 2005). Later human studies

93 showed increased HFO activity 10 seconds before seizure onset (Zijlmans et al. 2011) and

94 increased high-frequency power 8 seconds before seizure onset (Khosravani et al. 2009).

95 Another investigation analyzing fifteen minutes preceding seizures found preictal changes in

96 HFO rates and HFO-band power in all patients. However, the magnitude and direction of these

97 changes were variable, and there were no clear systematic trends across patients and seizures

98 (Jacobs et al. 2009). The majority of these analyses focused on counting the number of HFOs

99 and determining the peak spectral content to classify them as ripples or fast ripples. We

100 hypothesize that quantifying other features of HFO signals and evaluating them with more robust

101 statistics provides information critical to characterizing HFOs. This information could lead to

102 new algorithms capable of predicting seizures or identifying abnormal areas of brain tissue.

103

104 The current study evaluates HFOs as a temporal biomarker for seizures, analyzing 86,151

105 events from five patients with neocortical epilepsy. HFOs were detected using an automated

106 algorithm and grouped with unsupervised clustering (Blanco et al. 2010). This method allows 
107 for processing massive datasets in a manner that minimizes human bias (Gardner et al. 2007).

108 Using this dataset, we quantified waveform morphology using several signal processing features.

109 These features were the basis for comparing the dynamics of HFOs in the epochs surrounding

110 seizures, using several supervised classifiers and nonparametric statistical tests. The temporal

111 changes in the rates of each type of HFO were also evaluated. We find that each patient has

112 unique, statistically significant temporal changes in HFO rates and features in the 30 minute

113 period before and after seizures.

114

\section{Methods}

116 Patient Selection and Data Acquisition

117 We used a previously published HFO dataset (Blanco et al. 2010). Nine patients

118 diagnosed with medically refractory epilepsy were implanted with subdural electrodes (Ad-Tech

119 Medical Instruments, Racine, WI). As the goal of the analysis was to characterize HFOs at

120 different times relative to seizure onset, for inclusion in the study we required the presence of at

121 least one HFO during an electrographic seizure. Five of the nine patients met this study criterion.

122 Three of those excluded did not have any seizures, and the fourth did experience any HFOs

123 during the single seizure. Table 1 shows the clinical characteristics and electrode type for each of

124 the five included patients. The experimental protocol involved medication taper but not sleep 125 staging.

127 Electrodes were modified versions of standard grid and strip electrodes with added arrays 128 of non-penetrating, $40 \mu \mathrm{m}$ platinum-iridium "micro" wires (Van Gompel et al. 2008). Besides

129 intracranial electrodes, all patients received a limited montage of standard gold scalp electrodes, 
130 as well as other electrodes placed on the chin and anterior surface of the tibialis muscle to record

131 electromyographic activity. Stainless steel surgical sutures (Ethicon, Somerville, NJ) located at

132 the vertex region of the head served as the reference and ground for the intra- and extracranial

133 electrodes. Data were acquired on a Digital Lynx Data Acquisition System (Neuralynx,

134 Bozeman, MT) continuously at 32,556 samples per second with 18-bit resolution in up to 144

135 channels per patient, with a 9-kHz low-pass anti-aliasing filter.

HFOs were previously extracted from raw EEG data, as described in (Blanco et al.

138 2010). Briefly, we used a well-known HFO detector (Staba et al. 2002) to identify candidate

139 events, then clustered them using an unsupervised algorithm that did not presuppose the number

140 of subpopulations of HFOs. This algorithm identified four clusters of HFOs, corresponding

141 roughly to ripples, fast ripples, mixed events, and artifact. Events classified as artifacts were

142 removed, and all remaining detected HFOs in each patient were used in the analysis. It is

143 important to point out that the clustering algorithm removed filtering artifacts (Benar et al. 2010)

144 and other nonphysiological waveforms with a success rate that was similar to trained human

145 reviewers, who reviewed over 4700 individual HFOs in raw and filtered form. The algorithm

146 was used on the entire dataset without modification for ictal versus interictal period. The present

147 work is comprised of all HFOs that were detected and validated in the previous paper (Blanco et

148 al. 2010), excluding the artifacts. The EEG data, as well as the HFO markings, are freely

149 available at www.ieeg.org (Appendix A).

150

151 Feature extraction

152 Eight quantitative measures were calculated from each of the 86,151 remaining HFOs, a 
153 process known as feature extraction. The features were: 1) fast ripple/ripple band power ratio; 2)

154 spectral centroid; 3) spectral peak; 4) line length after spectral equalization; 5) bandpassed line

155 length; 6) zero-crossings per sample length; 7) maximum amplitude; and 8) number of peaks per

156 sample. The first four features characterize the frequency content of each event. The remaining

157 four features capture elements of waveform morphology that distinguish HFO classes in

158 published literature (see Appendix B). After calculating all features, we found that the data from

159 features 7 and 8 were highly correlated with others (correlation $>0.84$ ), but that the remaining 6

160 provided unique information. To reduce computational complexity, we used the reduced set of 6

161 features for all analyses except the initial classification (next paragraph).

162

163 HFO Ictal/Non-ictal Classification

164 We used three standard supervised machine-learning techniques to attempt to distinguish

165 between HFO events occurring during ictal versus non-ictal periods: logistic regression, k-

166 nearest neighbors (k-NN), and a support vector machine (SVM). Each of these techniques uses a

167 different algorithm to classify data. The goal is to determine how to label HFOs automatically in

168 subsequent data. We used two labels (ictal and non-ictal) in the first test to assess whether

169 classification was feasible, and if more complex classification experiments aimed at seizure

170 prediction would be possible. Information from all eight features was used to inform the

171 classifiers, and we performed Principal Components Analysis (PCA) to lessen the computational

172 burden on the learning algorithms. This reduced the eight features to five components while

173 retaining $96.3 \%$ of the data variance. We ran the experiments on the aggregate patient data and

174 on an individual patient basis. However, it is important to note that conclusive analyses were

175 performed on the full feature set, rather than just PCA data (see below). We split each set of 
176 samples into equally-sized sets of training and testing data.

To further simplify the initial test of the classifiers, we first trained them on data from

179 each patient individually, with a segregated partition of testing data. We also trained and tested

180 on data from all patients in aggregate. In each case, we created ten random partitions from the

181 training and testing data sets, reserving $25 \%$ of the samples in each data set for cross-validation.

182 Appendix $\mathrm{C}$ describes the details of the cross-validation for the three methods. We assessed

183 classifier performance on the testing data using the $F_{1}$ measure (Eq. 1), the harmonic mean of

184 sensitivity and precision:

185

$S=\frac{T P}{T P+F N}$

186

$$
P=\frac{T P}{T P+F P}
$$

$$
F_{1}=\frac{2 * S^{*} P}{S+P}
$$

(S: sensitivity, P: precision, TP: true positive, TN: true negative, FP: false positive)

We used a permutation test to compare the testing set results to those generated by randomly assigned ictal/non-ictal labels. This tested the null hypothesis that the classification was no better

191 than randomly assigning the labels. After permuting the labels of the training data, we retrained 192 the classifiers, then reclassified the testing data. We repeated this procedure for 10,000 trials for 193 each patient and for 10,000 trials of the aggregate of all five patients. We compared the original $194 \quad F_{1}$ performance of the classifiers to the expected performance under the null hypothesis (the 195 distribution of $F_{1}$ from the random permutations) at a significance level $\alpha=0.05$ (Bonferroni 196 corrected $\alpha=0.001$ ). 
After calculating all features, each HFO can be represented as a unique point in "feature

200 space" that has as many dimensions as the number of features. We divided the HFOs into four

201 time epochs: preictal, ictal, postictal, and interictal. Pre- and postictal windows were defined as

20210 minutes before and after a seizure, respectively. Interictal was defined as greater than 10

203 minutes from a seizure for this test. We tested whether the distribution of HFOs within the

204 feature space in each epoch was non-random. The null hypothesis was that the scattered

205 distribution of features of all HFOs in each epoch was no different than if the HFOs were

206 randomly assigned to an epoch. The centroid of features 1-6 was calculated (7-8 were not

207 included due to high correlation with the other features). We measured the Euclidean distance of

208 each HFO's features to the centroids, grouped HFOs according to epoch, and used the median

209 distance as the measure of dispersion for that epoch. We first calculated the dispersion using the

210 original labels. We then randomly permuted the epoch labels, determined a new centroid for each

211 of the four epochs, and recalculated the dispersion of all relabeled HFOs within that epoch,

212 repeating this procedure over 10,000 trials. The probability of the null hypothesis (i.e. that the

213 observed dispersion in each epoch was that of a random sample of all HFOs) was the proportion

214 of permutations with dispersion values more extreme than the real data, tested with a

215 significance level of 0.05 . Similar analyses were done with pre/postictal periods of several other 216 durations from 2-120 minutes; results of this analysis were similar.

218 Temporal evolution of HFO features

219 We assessed changes in the morphology features before and after seizures using two non220 parametric statistical tests that are resistant to outliers and/or skewed data. We first performed 
221 Principal Components Analysis (PCA) in each patient to reduce the set of HFO features to two

222 dimensions for the purpose of visualization. We divided the four epochs above into smaller 5- to

223 10-minute "stages" (10 total) between 0-30 minutes before and after seizures. Interictal for these

224 analyses was defined as greater than 30 minutes from a seizure. We made scatter plots of the

225 HFOs in the first two PCA dimensions for each stage. The Kruskal-Wallis Test of the first two

226 PCA components determined whether the 2-dimensional PCA distributions from the different

227 stages were unique versus samples of the same distribution. We then evaluated temporal changes

228 of the six individual morphology features (see previous paragraph) using Spearman's rank

229 correlation, the nonparametric equivalent of the Pearson correlation. This test, which used 6

230 features explicitly, served as a more robust analysis of the conclusions in the PCA data. This test

231 determines whether there is a monotonic relationship between time and each feature. HFOs prior

232 to the first seizure or after the last were excluded from this analysis, as their relationship to

233 previous or subsequent seizures could not be determined.

235 Stereotyped responses in different seizures

236 We evaluated whether the temporal changes above were consistent from seizure to

237 seizure within each patient with an analysis of variance (ANOVA) on the first two PCA

238 components in each of the ten stages. We calculated the variance of each component in each

239 temporal stage for each seizure, then computed the ANOVA across all seizures in each patient.

240 The ANOVA p-values were averaged across each patient and across each stage: high p-values

241 indicate that the distributions are similar from seizure to seizure, i.e. that the response is

242 stereotyped. 
In order to analyze temporal changes in the rates and proportions of each of the three

246 HFO classes (ripples, fast ripples, mixed events (Blanco et al. 2010)), we calculated the average

247 rate (events/minute) and percentage of total events from each HFO class within each of the 5- to

248 10-minute stages described above. To quantify the relationship between HFO class and latency

249 to seizure, we performed a Kruskal-Wallis test comparing the rank order of each class with time

250 to nearest seizure, aggregating all seizures. This was done twice, evaluating time to next and time

251 to previous seizure. A significant p-value $(<0.05)$ indicates that the three clusters have different

252 distributions, in other words that each class tends to occur at different times in relation to the

253 nearest seizure. We tested whether the preictal rates of each class change significantly from

254 interictal baseline with a chi-square test, comparing the proportion of each type of HFO during

255 interictal versus the combination of all preictal stages (0-30 minutes).

$257 \quad$ Results

258 HFO Ictal/Non-ictal Classification

259 The first analysis tested three classifiers to determine whether they could distinguish

260 HFOs occurring during seizures (ictal) from those occurring at other times (non-ictal, including

261 interictal, postictal, and preictal). Figure 1 shows how well each classifier was able to separate

262 the morphology of ictal and non-ictal HFOs, which we quantified using the $\mathrm{F}_{1}$ measure. The

263 logistic regression classifier is not included in the figure because it did not make a single true

264 positive identification. A higher $\mathrm{F}_{1}$ score indicates a larger number of true positives relative to

265 false positives and false negatives. The highest possible score is 1, resulting from perfect

266 sensitivity and precision. The classifiers had limited success in separating the ictal and non-ictal 
267 HFOs. This is in part due to the fact that the proportion of non-ictal HFOs was much greater than

268 ictal in the training data. The k-NN and SVM classifiers were statistically superior to random

269 classification (0.05 significance, Bonferroni correction $\mathrm{p}<<0.001)$ in patients $\mathrm{A}$ and $\mathrm{B}$, and the

$270 \mathrm{k}-\mathrm{NN}$ was superior to random classification in patient D and in the aggregate of all 5 patients.

271 Thus, in certain patients it was possible to distinguish ictal from non-ictal HFOs better than

272 random; however, the actual F1 scores were all $<0.2$, suggesting the classification was not very

273 sensitive nor precise.

274

275 Temporal distribution of HFO features

276 To evaluate whether any feature characteristics varied over time, we further subdivided

277 the non-ictal period into postictal, preictal, and interictal epochs. Table 2 displays the number of

278 HFOs in each epoch. Seizures were a mean of 120 minutes apart (range 9.5 to $839 \mathrm{~min}$ ). In three

279 patients, some seizures occurred less than one hour apart, causing pre- and postictal periods to

280 overlap. HFOs occurring during these overlaps were counted as both pre- and post-ictal events,

281 which may introduce bias into some of the following analyses. However, this involved a small

282 number of HFOs ( $15 \%$ of HFOs in patient $\mathrm{A}$, none in $\mathrm{B}$ and $\mathrm{C}$, and $1 \%$ in $\mathrm{D}$ and $\mathrm{E}$ ), and

283 removing them from analysis did not change any of the conclusions (next section). In each

284 patient, we tested whether the distribution of HFOs in feature space was different during each

285 epoch than what would be expected from a random sample of HFOs from all epochs in that

286 patient. Figure 2 displays a plot of all HFOs from one patient, projected into two-dimensional

287 feature space via PCA, with raw data from several HFOs displayed to demonstrate the varied

288 appearance across the PCA space. Figure 3 displays the same data separated into time epochs,

289 showing that the HFO distribution in each epoch (i.e. the "shape" of the scattered HFOs) differs 
290 from the shape of the population as a whole. Similar findings were found in the other patients, 291 though the distributions and rates of each HFO type were very different from this patient (see

292 Fig. 6). Though it cannot be displayed graphically, the HFO distributions using all six features

293 have similar temporal differences. When analyzing the data in six dimensions (one for each

294 feature), the distributions were significantly different than random: there were specific

295 characteristics during the different epochs, demonstrated by a difference in cluster dispersion as

296 measured by Euclidean distance $(\mathrm{p}<0.05)$. We made similar temporal comparisons for each

297 feature individually and found great inter-patient variability. Table 3 shows the p-values for two

298 example features, the line length after spectral equalization and power band ratio. The other

299 features demonstrated similar inter-patient variability and were significant in some, but never all,

300 of the five patients. These results imply that HFO distributions vary during different peri-ictal

301 epochs and involve complex relationships between several features, but that these changes are

302 unique to individual patients. Thus, samples from different times, especially across different

303 patients, will likely have different feature distributions.

304

305 Temporal evolution of HFO features

306 The previous section tested whether each epoch had a different distribution from random.

307 We next sought to test the time-dependency of HFO features. The first step was to compare the

308 distributions in each stage in the 2-dimensional PCA plots (e.g. Fig. 3 for Patient C). The

309 Kruskal-Wallis Test demonstrated that for all patients there were highly significant differences

310 between stages $(\mathrm{p}<<0.0001)$. We then evaluated how the individual features changed

311 temporally, using time before or after seizure as a continuous variable rather than constraining

312 the analysis to categorical epochs. We used the Spearman correlation to assess whether there was 
313 any time dependence for each of the six individual features. The Spearman correlation

314 determines strength and direction of any monotonic relationship between a feature and time; in

315 this case, it assesses how much of a measureable, progressive change the HFO features have as a

316 function of time prior or subsequent to seizure. Figure 4 shows the Spearman correlations for

317 each patient. Correlations closer to \pm 1 indicate stronger relationships. In most cases, several

318 features demonstrated weak but statistically significant correlations with both time to next

319 seizure and time from previous seizure. Interestingly, certain features showed a significant

320 positive correlation with time in some patients and a significant negative correlation with time in

321 others. These analyses were repeated in patients A, D, and E with the overlapping HFOs

322 removed, and there were no substantive changes to the conclusions: Kruskal Wallis tests were

323 still all significant, and there were minor changes to the Spearman correlations (Fig. 4B).

324 In order for these temporal changes to be helpful in the development of customized

325 seizure prediction algorithms, they must be consistent from seizure to seizure. Looking at each

326 patient individually, we evaluated the seizure to seizure variability by running ANOVA on the

327 first two PCA components in each peri-ictal stage across all seizures. In this analysis, when the

328 p-value is "insignificant" ( $>0.05)$, it corresponds to a similar distribution of the PCA values in

329 subsequent seizures, i.e., the response is stereotyped and thus the ANOVA fails to find a

330 difference between them. Conversely, a "significant" p-value indicates there are differences

331 across seizures, so the distributions of PCA are not stereotyped. An example of each case is

332 shown in Fig. 5. These plots demonstrate that some patients are stereotyped while others are not,

333 but do not capture all of the temporal changes described in previous paragraphs. The HFO

334 distributions are quite similar from different seizure periods for example, there are a large

335 number of HFOs 20-30 minutes before seizure 3 with different morphology, so it would be 
336 difficult to predict seizure onset in this patient based upon these data. We calculated the ANOVA

337 of the PCA distributions across different seizures in every stage for every patient to determine if

338 the changes were stereotyped. We found that three of the patients (A, B, E) had stereotyped

339 responses across all stages ( $\mathrm{p}>0.05$ ) (Fig. 5C). In the other two patients (C, D), the ANOVA

340 found that at least one of the PCA components was significantly different between different

341 seizures, meaning it would be hard to predict how the distribution would look in later seizures.

342 Taken together, these data demonstrate that several HFO features are correlated with time, that

343 these changes are often stereotyped, but that there is significant inter-patient variability.

\section{Temporal evolution of HFO rate and class}

Although it is clear from these analyses that a wide set of features is helpful to

347 describe HFOs and their temporal dynamics, the data also suggest that the relative rate of each

348 HFO cluster (ripple, fast ripple, mixed event) may differ between different stages. Constraining

349 each HFO to one of 3 cluster labels removes most information about its dynamic features, but is

350 also the most common way they are currently studied. In order to evaluate any changes

351 descriptively, we first plotted the relative rates of each HFO cluster, as well as the total HFO

352 rate, for each patient in each stage (Fig. 6). We observed distinct patient-specific trends in the

353 evolution of rates and proportions of each cluster before and after seizures. In three of the

354 patients (A, B, D), the total number of HFOs is relatively constant until an increase during the

355 ictal period, an effect less pronounced in patient B. But the individual HFO clusters behave quite

356 differently in these three patients. In A, there is a subtledrop in the proportional of ripples just

357 before the seizures. B has a similar effect postictally. In D, mixed events dominate preictally,

358 but disappear during the seizures. Observing the datapoints from individual seizures shows that 
359 similar findings are seen at each seizure. Patients $\mathrm{C}$ and $\mathrm{E}$ have much smaller changes in total

360 HFO rate, sometimes having fewer HFOs during seizures. The only consistent change in patient

$361 \mathrm{C}$ is increased mixed events and loss of ripples during seizures. Patient $\mathrm{E}$ is difficult to evaluate

362 with only 2 seizures, but tends to have a predominance of ripples preictally and mixed events

363 immediately postictally. We tested whether the HFO rates changed significantly from baseline

364 by comparing the proportion of each HFO cluster in the interictal stage with the aggregate of the

3654 preictal stages. Patients C, D and E had very significant changes from baseline, while A and B

366 did not $(\mathrm{p}<<0.0001$, chi-square).In order to quantify the relationships between each type of HFO

367 and time to seizure in Fig. 6, we compared the firing times of every HFO with a Kruskal-Wallis

368 test using time to the previous and subsequent seizure as continuous variables. This assesses by

369 rank order whether there are trends in the temporal distribution of each HFO cluster in each

370 patient. A significant result $(\mathrm{p}<0.05)$ indicates that the firing times of the three clusters are

371 unlikely to be randomly distributed - that at least one type of HFO (ripple, fast ripple, mixed

372 event) is more likely to occur at specific times between seizures. We found that HFO times both

373 before and after seizures were significantly different in all patients and times (Table 4, Kruskal-

374 Wallis Test, $\mathrm{p}<0.05$ ). Thus, in each patient specific types of HFOs became more or less likely to

375 occur in the pre- and post-ictal periods. These findings demonstrate the strong temporal

376 relationships HFOs have with seizures as well as the disparate patterns found in different

377 patients.

378

379 Discussion

380 Peri-ictal HFO distributions vary greatly among patients

381 Our current analysis evaluates properties of three classes of HFOs in four epochs (inter-, 
382 pre-, postictal, and ictal), collecting a large number of events in 5 patients. This allowed a

383 thorough comparison of the statistical characteristics of HFOs during these epochs. Our use of

384 three classes of HFO is a slight departure from previous work on these types of oscillations in

385 patients with epilepsy. The first two classes are very similar to ripple and fast ripples, which are

386 commonly recognized in the literature and were originally identified based upon their peak

387 oscillation frequency. In this work, we have included mixed events that were identified by an

388 unsupervised algorithm (Blanco et al. 2010) as a third class, as their power spectra contain

389 features of both ripples and fast ripples

390

391 Our results show that the relative and absolute rates of each class of HFO changed in the

392 periods before and after seizures, and that these effects varied greatly among patients (Fig. 6).

393 The ictal period was most variable in terms of the number and type of HFOs. The total HFO rate

394 increased in three of the patients during seizures, while in the other two patients it decreased in

395 the preictal period. Such disparities in preictal HFO rates have been identified previously

396 (Jacobs et al. 2009); to our knowledge this is the first work evaluating the postictal and interictal

397 periods as well. Interestingly, not all patients exhibited their highest total HFO rate during

398 seizures. Fast ripple and mixed event rate increased beginning from 20-30 minutes prior to

399 seizures in all patients but B. Fast ripple rates increased postictally in patients B-E. Ripples were

400 predominant in 3 patients, though the ratio of ripples to fast ripples changed significantly in the 5

401 minutes before and after seizures. The time between seizures was highest in patients B and C,

402 but there are no clear patterns that distinguish these two patients from the others. It is notable

403 that there were no trends consistent among all 5 patients. With such a small patient sample size

404 identifying so many differences between individuals, it is likely a common occurrence for 
405 patients to have unique HFO distributions. The cause of these findings is not clear. There are two

406 main possibilities. The first is that behavior of HFOs during, between and after periods of

407 seizure generation may be unique to a patient's epileptic network or mechanism of epilepsy. Our

408 analysis of PCA features (Fig. 5) revealed that at least three of the patients had unique responses

409 that were stereotyped in different seizures, suggesting this may be true in some patients. Another

410 possibility is that these individualized patterns may be due in part to sampling error, as there is

411 no uniform implant strategy for all patients, and the microwires which detected many of the

412 HFOs are dispersed unevenly in the implanted grids. These questions could be explored further

413 by a larger sample with different implantation strategies that maximize high resolution electrode

414 coverage in the brain, or perhaps by guiding electrode placement through intraoperative mapping

415 of HFO generating regions to augment standard electrode placement strategies. Whatever the

416 cause, our findings suggest that each patient must be evaluated individually when using HFO

417 rates as an electrical seizure biomarker.

418 One intriguing possibility for future work lies in comparing the results from patients B

419 and D, which had the most similar ictal responses in Fig. 6 (increased rate of HFO and ripples

420 during seizure). These two patients both had frontal cortical dysplasia (Table 1). Although this

421 sample size is small, this result suggests that future work should aim to stratify patients by

422 epileptic pathology, and perhaps common trends could be identified. Adding patients with other

423 types of epilepsy (e.g. mesial temporal sclerosis) would also provide crucial information. Such

424 work would likely also benefit from additional features that were not present in the current

425 analysis.

426

427 HFO features during the ictal period, and seizure prediction 
We tested three machine learning algorithms with a large dataset of automatically

429 detected HFOs to determine whether supervised classification could be used to identify

430 differences between ictal and nonictal HFOs. Using the measurements described in Appendix B,

431 none of the algorithms obtained an F1 score higher than 0.2, which is fairly poor performance.

432 This finding suggests that, at least with these tools and features, it is difficult to distinguish any

433 HFO signal features that are specific to the ictal period.

434 Despite the low performance, two methods did beat random chance, so we further

435 investigated the temporal changes in HFOs in the peri-ictal period to determine the source of the

436 differences. The first step was to analyze the HFO features in "feature space." When the HFOs

437 were grouped together based upon stage, each patient's HFO distribution had a unique shape in

438 feature space that changed over time. We found that these changes continued to evolve as much

439 as 30 minutes before or after seizures. We tested these changes in several different ways:

440 comparing the distributions of their PCA-reduced features, comparing their 6-dimensional

441 distributions to the distributions of random samples, and determining the Spearman correlation

442 of each feature with time before and after seizures. In each case, there was strong statistical

443 evidence of measureable temporal changes in HFO features.

444 These findings have significant implications for developing automated seizure prediction

445 or warning algorithms. Although this analysis did not attempt to develop such an algorithm, it

446 was performed on an unbiased sample of continuous EEG, thus simulating the "real-world"

447 conditions necessary for such an algorithm to be successful. Additionally, it is well known that

448 classification algorithms used for seizure detection and prediction usually perform better with

449 input from multiple orthogonal features; most of our analyses were based upon single or PCA-

450 reduced features. Future attempts to develop seizure prediction algorithms based upon 
451 combinations of these and other features may be very useful, but will need to be rigorously tested

452 using independent testing and training sets with prolonged continuous intracranial EEG,

453 comparing against chance predictors using established methods (Mormann et al. 2007; Snyder et

454 al. 2008). Furthermore, these data also suggest that these algorithms will be most effective if

455 they are personalized for individual patients based upon their unique features (Stacey and Litt

456 2008). For example, certain features might be relevant for one patient but not another, or the

457 correlation of certain features with seizures might differ in strength or direction between patients.

458 This will require a thorough analysis of HFO data on a per-patient basis before adjusting and

459 applying the prediction algorithm. It will be critical to identify appropriate patients for such

460 algorithms, and likewise not to abandon these techniques if they fail in some patients.

Identifying abnormal, epileptic HFOs

463 As HFOs have been identified in both normal and abnormal brain, it is imperative that

464 any clinical decisions based upon them are able to distinguish whether they are markers of

465 abnormal activity or not (Engel et al. 2009). To date, the primary clinical strategies proposed are

466 to identify the electrodes with the highest number of HFOs (Jacobs et al. 2010) or to stratify

467 HFOs into different categories based upon peak frequency (i.e. ripples $100-250 \mathrm{~Hz}$, fast ripples $>$

$468250 \mathrm{~Hz}$ ) (Akiyama et al. 2011). While such strategies are promising, they were based upon

469 selected retrospective data, and there is considerable risk in basing prospective clinical decisions

470 upon such data. The primary concern is that HFOs also occur outside the seizure onset zone,

471 even in patients who do not have epilepsy at all (Blanco et al. 2011). It is quite possible that any

472 patient can have a region with a relatively higher HFO density, but that the increase could be due

473 to local edema, physiological variation, or various other non-epileptic causes. It is therefore 
474 crucial to develop a method of characterizing HFOs that are true markers of epileptic tissue.

475 One characteristic of HFOs that has received relatively little attention is their temporal 476 variation. A recent study evaluated HFOs and sharp waves with respect to seizures and found 477 that, unlike sharp waves, HFO incidence changes similar to seizures (Zijlmans et al. 2009). This 478 finding is intriguing in light of recent work investigating the mechanisms of abnormal HFOs and 479 seizures. Ripples were originally described in normal tissue (Ylinen et al. 1995), and are 480 considered to be the product of fast synchronous firing of inhibitory potentials. Conversely, later 481 work in epileptic tissue has shown that "abnormal" HFOs are comprised of population spikes 482 (Bragin et al. 2011). This difference has led to a great deal of research to determine how 483 epileptic HFOs are made (Jefferys et al. 2012). But detailed physiological studies into these 484 phenomena are extremely difficult with current technology. Therefore, computational modeling 485 has been used to confirm the role of population spikes in ripples (Stacey et al. 2011; Stacey et al. 486 2009; Wendling et al. 2012) and fast ripples (Demont-Guignard et al. 2012; Ibarz et al. 2010; 487 Roopun et al. 2010). In each case, epileptic pathologies (i.e. increased excitation, decreased 488 inhibition, abnormal coupling) cause the abnormal HFO to occur. Wending et al. demonstrated 489 that HFO characteristics change on a continuum from normal activity to seizure, depending upon 490 the parameters (Wendling et al. 2012). These realistic modeling studies suggest that epileptic 491 pathologies will alter the appearance of HFOs, and predict that a wide range of parameters 492 (Demont-Guignard et al. 2012; Stacey et al. 2009) can be responsible. Our data demonstrate 493 clinical evidence supporting this: that there are temporal changes in HFO morphology leading 494 into seizures. These data likely represent a change in underlying physiology that is not yet 495 quantified, which suggests that a robust feature analysis of HFOs may be able to illuminate 496 future research into the underpinnings of this phenomenon. 
If one assumes that there is an underlying process that alters the network parameters

498 before and after seizures, and that it affects both seizures and HFOs, it follows that HFOs may

499 have different features in the time surrounding seizures. Until technology is able to characterize

500 these network phenomena on smaller scales, our best method is to analyze EEG data. In this

501 study, we analyze an unbiased sample of intracranial EEG and find that there are indeed

502 temporal changes in HFO characteristics. However, the differences were relatively small and

503 were highly variable between patients. Further work evaluating different signal features,

504 recording EEG on different spatial and temporal scales, and comparing HFOs from "normal" and

505 "abnormal" regions may be better able to distinguish these changes and lead to understanding of

506 the mechanisms involved in generating both HFOs and seizures.

\section{Conclusion}

509 With increasing interest in and evidence for HFOs as an electrical seizure biomarker, it is

510 crucial to develop automated methods to identify and classify them that are not operator

511 dependent, and that can be applied to a wide range of patients as well as animal models. Using

512 data from an automated HFO detection algorithm, this study provides statistical evidence that

513 some patients demonstrate temporal changes in the distribution of HFOs in the periods before

514 and after seizures. Initially, these findings can be the basis of further experimental algorithms to

515 explore temporal phenomena such as seizure generation, prediction, and epileptogenesis. With

516 further validation, they may also provide a basis for algorithms capable of identifying periods of

517 increased risk of seizure onset. Perhaps the most important finding from this study is that

518 individual patients have vastly different HFO patterns related to seizures; these findings suggest

519 that any clinical decision based upon HFO analysis must be customized to each individual 
520 patient.

521

522 


\section{Figure Legends}

525 Figure 1: Classifier performance for the aggregate patient data and for individual patients as

526 measured by the $\mathrm{F}_{1}$ score, the harmonic mean of sensitivity and precision $(\max =1)$. Statistical

527 significance at the level of $\alpha=0.05$ (Bonferroni corrected $\alpha=0.001$ ) is indicated by an asterisk.

528 Results from the logistic regression classifier are not shown because the $\mathrm{F}_{1}$ score was zero in

529 every trial. SVM: support vector machine; k-NN: k-Nearest Neighbor.

531 Figure 2: Scatter plot of all HFOs in Patient $\mathrm{C}$ in the first two dimensions of the PCA space.

532 Each dot represents one HFO and is colored according to HFO cluster as determined by (Blanco

533 et al. 2010). Localization of each dot corresponds to the first and second PCA components

534 plotted in x-y coordinates. Representative waveforms from different regions of the PCA feature

535 space provide examples of the morphology at each location.

537 Figure 3: Outside: Scatter plots of the HFOs in each time stage for Patient $\mathrm{C}$ in the first two

538 dimensions of the PCA space. Color indicates HFO cluster as in Fig. 2. Bar graphs show the 539 average rate (events per minute) for each cluster. Center: Scatter plot of all HFOs from Patient C.

541 Figure 4: A: Bar graphs showing the Spearman correlations for morphology features and time to 542 next seizure (left) and time from previous seizure (right). Statistically significant correlations $(\alpha$ $543=0.05)$ are indicated by an asterisk. B: Repeat analysis when overlapping HFOs are removed.

544 Patient B and $\mathrm{C}$ had none so the analysis was unchanged (italics). Black circles indicate subtle

545 differences from A, most notably that two values in patients A and D become significant $546(\mathrm{p}<0.05)$. Kruskal Wallis tests all remained $\mathrm{p}<0.001$ when overlappers were removed (not 
547 shown).

550 Figure 5: Temporal HFO distributions are stereotyped across different seizures in some patients.

551 HFOs are displayed by their first two principle components and stratified by seizure. Plot is

552 similar in organization to Fig. 3, but coloring corresponds to nearest seizure. A: In patient C,

553 there are distinct patterns seen before or after certain seizures, such as 30-20 minute preictally in

554 seizure 3. This patient does not have stereotyped HFO distributions $(\mathrm{p}<0.05)$. B: Patient A has

555 very similar distribution across all seizures in every stage, a stereotyped response ( $>0.1)$. All

556 plots have same axes and scale, shown in lower left (bars: 1 unit). C: P-values for the ANOVA

557 of the first two PCA components. Bar graphs of average p-value across all stages except

558 interictal in each patient. Bars indicate one standard deviation ( $\mathrm{n}=9$ stages). Asterisks indicate

559 statistical significance $(\mathrm{p}<0.05)$ Insignificant $\mathrm{p}$-values correspond to similarity in the

560 distributions.

563 Figure 6: Graphs of the relative rates of HFOs in each frequency cluster (orange, blue, and pink 564 lines; left axis) and the total HFO rate (dotted dark line; right axis). Rates of each HFO cluster 565 during individual seizures are indicated with indicated symbols; lines indicate mean across all 566 seizures. Error bars: standard deviation of total rate. Lack of any consistent trends among 567 different patients implies that there is marked inter-patient variability in HFO rates before and 568 after seizures. Clustering of datapoints from different seizures indicates stereotyped responses, 569 most prominent in patients A, B and D. \#- Chi-square testing indicated that proportions of each 
570 HFO cluster are significantly different from interictal in the patients C, D, and E. Parentheses

571 next to patient name indicate number of seizures in each patient.

572

573 


\begin{tabular}{|c|c|c|c|c|c|}
\hline Subject & Age & Sex & Pathology & Electrodes & Electrode Placement \\
\hline $\begin{array}{c}\mathrm{A} \\
(\mathrm{SZ} 01)\end{array}$ & 35 & $\mathrm{~F}$ & $\begin{array}{l}\text { Frontal neocortical } \\
\text { oligodendroglioma }\end{array}$ & $\begin{array}{l}\text { Standard: } 6 \times 6 \\
\text { Micro: } 1 \times 8\end{array}$ & Right: frontal, motor cortex \\
\hline $\begin{array}{c}\mathrm{B} \\
(\mathrm{SZ} 02)\end{array}$ & 24 & M & $\begin{array}{l}\text { Frontal cortical } \\
\text { dysplasia }\end{array}$ & $\begin{array}{l}\text { Standard: } 6 \times 6 \\
\text { Micro: } 4 \times 6\end{array}$ & Left: frontal, frontoparietal \\
\hline $\begin{array}{c}\mathrm{C} \\
(\mathrm{SZ} 04)\end{array}$ & 39 & $\mathrm{~F}$ & $\begin{array}{l}\text { Temporal } \\
\text { neocortical gliosis }\end{array}$ & $\begin{array}{l}\text { Standard: } 6 \times 6,1 \times 8 \\
1 \times 8,1 \times 4,1 \times 4,1 \times 4 \\
\text { Micro: } 1 \times 4\end{array}$ & $\begin{array}{l}\text { Left: temporal, inferior frontal, superior } \\
\text { frontal, anterior temporal, posterior } \\
\text { temporal, anterior, posterior }\end{array}$ \\
\hline $\begin{array}{c}\mathrm{D} \\
(\mathrm{SZ} 07)\end{array}$ & 42 & $\mathrm{~F}$ & $\begin{array}{l}\text { Frontal cortical } \\
\text { dysplasia }\end{array}$ & $\begin{array}{l}\text { Standard: } 6 \times 8,3 \times 8 \\
1 \times 4 \\
\text { Micro: } 1 \times 4,1 \times 8\end{array}$ & $\begin{array}{l}\text { Right: frontal, temporal, anterior } \\
\text { temporal, inferior temporal, posterior } \\
\text { temporal }\end{array}$ \\
\hline $\begin{array}{c}\mathrm{E} \\
(\mathrm{SZ} 08)\end{array}$ & 38 & $\mathrm{~F}$ & $\begin{array}{l}\text { Temporal } \\
\text { neocortical gliosis }\end{array}$ & $\begin{array}{l}\text { Standard: } 6 \times 6 \\
\text { Standard depth: } \\
1 \times 4 \\
\text { Micro depth: } 1 \times 4 \text {, } \\
1 \times 4\end{array}$ & $\begin{array}{l}\text { Left: temporal, anterior temporal, } \\
\text { middle temporal, posterior temporal }\end{array}$ \\
\hline
\end{tabular}

578 Summary of patient clinical characteristics and electrode placement. Parentheses: labels used in

579 prior study, see Table 1 in (Blanco et al. 2011) for further details. 
583 Table 2: HFO and seizure counts

584

\begin{tabular}{|c|r|r|r|r|r|r|}
\hline Patient & $\begin{array}{c}\text { Total } \\
\text { seizures }\end{array}$ & $\begin{array}{c}\text { Total } \\
\text { HFOs }\end{array}$ & $\begin{array}{c}\text { Inter- } \\
\text { ictal }\end{array}$ & Preictal & Ictal & $\begin{array}{r}\text { Post- } \\
\text { ictal }\end{array}$ \\
\hline A & 9 & 2552 & 1487 & 314 & 371 & 380 \\
\hline B & 3 & 9905 & 9237 & 280 & 139 & 249 \\
\hline C & 4 & 12417 & 11585 & 329 & 317 & 186 \\
\hline D & 7 & 53015 & 49519 & 1787 & 433 & 1276 \\
\hline E & 2 & 8262 & 7210 & 493 & 46 & 513 \\
\hline All & 25 & 86151 & 79038 & 3206 & 1306 & 2604 \\
\hline
\end{tabular}

586 Counts of HFOs in each time epoch for dispersion analysis. Preictal and postictal epochs were

587 defined as 10 minutes before and after seizures, respectively.

588

589 
591 Table 3: Dispersion p-values from 2 features

592

\begin{tabular}{|l|c|r|r|r|r|r|}
\cline { 2 - 7 } \multicolumn{1}{c|}{} & \multicolumn{6}{c|}{ Feature 3 (Line length) } \\
\cline { 2 - 7 } \multicolumn{1}{c|}{} & Pt A & Pt B & Pt C & Pt D & Pt E & \multicolumn{1}{c|}{ All } \\
\hline Interictal & 0.0018 & $*$ & $*$ & $*$ & 0.0011 & $*$ \\
\hline Preictal & $\mathbf{0 . 3 7 1 3}$ & $\mathbf{0 . 0 6 1 5}$ & $*$ & $*$ & 0.0002 & $\mathbf{0 . 2 2 4 2}$ \\
\hline Ictal & 0.0426 & 0.0007 & $*$ & $\mathbf{0 . 3 1 4 8}$ & 0.0155 & $*$ \\
\hline Postictal & $\mathbf{0 . 0 9 8 3}$ & $*$ & $*$ & 0.0038 & $*$ & $*$ \\
\hline
\end{tabular}

593

\begin{tabular}{|l|r|c|r|r|r|c|}
\cline { 2 - 7 } \multicolumn{1}{c|}{} & \multicolumn{6}{c|}{ Feature 4 (Power Band Ratio) } \\
\cline { 2 - 7 } \multicolumn{1}{c|}{} & Pt A & Pt B & Pt C & Pt D & Pt E & \multicolumn{1}{c|}{ All } \\
\hline Interictal & $*$ & $\mathbf{0 . 2 2 8 5}$ & $*$ & $*$ & $*$ & $\mathbf{0 . 1 3 9 8}$ \\
\hline Preictal & 0.0442 & $\mathbf{0 . 4 9 1 6}$ & $*$ & $*$ & $\mathbf{0 . 4 2 0 8}$ & $*$ \\
\hline Ictal & $*$ & 0.0001 & $*$ & $*$ & $*$ & $*$ \\
\hline Postictal & $*$ & $\mathbf{0 . 1 0 5 9}$ & $*$ & $*$ & $*$ & $\mathbf{0 . 3 2 8 5}$ \\
\hline
\end{tabular}

Table 3: P-values from the epoch dispersion analysis. Values were calculated by dividing the

597 number of permutation test trials with results more extreme than the true values by the total

598 number of permutation test trials $(10,000)$. Bold text indicates values that were not statistically

599 significant. The combination of all (six) features was significant in all patients. Each patient had

600 different trends with the individual features. Asterisks: highly significant $(\mathrm{p}<1 \mathrm{e}-6)$

601

602 
603 Table 4: P-values of temporal rank

604

\begin{tabular}{|l|l|l|l|}
\cline { 2 - 4 } \multicolumn{1}{c|}{} & $\begin{array}{l}\text { Time to } \\
\text { Next }\end{array}$ & $\begin{array}{l}\text { Time From } \\
\text { Previous }\end{array}$ & $\begin{array}{l}\text { Closest } \\
\text { cluster (next) }\end{array}$ \\
\hline Pt A & $<.0001^{*}$ & $<.0001^{*}$ & FR, M \\
\hline Pt B & $0.013^{*}$ & $<.0001^{*}$ & M, R \\
\hline Pt C & $<.0001^{*}$ & $<.0001^{*}$ & FR, R \\
\hline Pt D & $<.0001^{*}$ & $<.0001^{*}$ & FR, R \\
\hline Pt E & $<.0001^{*}$ & $<.0001^{*}$ & R \\
\hline
\end{tabular}

605

606

607 Table 4: P-values from the second Kruskal-Wallis Test, used to assess whether an HFO near a

608 seizure was more likely to be of a specific cluster. Third column indicates which clusters were

609 closest to the next seizure (R: ripple, M: mixed, FR: fast ripple ). Asterisks: statistical

610 significance.

611

612 


\section{Appendix A: Online access to data at www.ieeg.org}

614 The EEG data and HFO markings used in this study have been posted by the authors of (Blanco

615 et al. 2011) for viewing and analysis on the IEEG-Portal (https://www.ieeg.org). This free online

616 database allows the user to view, download, and annotate EEG data, and has been established to

617 allow datasharing and collaboration. The five studies used in this manuscript can be accessed

618 with the following names:

619 Patient A- I001_P034_D01; Patient B- I001_P011_D01; Patient C- I001_P015_D01; Patient

620 D- I001_P014_D01; Patient E- I001_P017_D02.

621

622

623

624

625

626

627

628

629

630

631

632

633

634

635

636

637

638

\section{Appendix B: Feature Equations}

1) Power Band Ratio $(250-500 \mathrm{~Hz}) /(100-200 \mathrm{~Hz})$ : The ratio of estimated power in the hypothesized "fast-ripple" band to that in the hypothesized "ripple" band. Computed on the band-passed data; frequency-input domain.

$$
\text { Power band ratio }=\widehat{\mathrm{P}}_{[250,500]} / \widehat{\mathrm{P}}_{[100,200]}
$$

where

$$
\hat{P}_{[a, b]}=\sum_{\{k \in Z \mid \zeta(a) \leq k \leq \zeta(b)\}}\left|M_{k}\right|^{2},(a<b) \in\left(0, \frac{F_{s}}{2}\right)
$$

and $M_{k}$ is a multitaper power spectral density estimate. For more details, see Blanco et al. 2010.

2) Spectral Centroid: The frequency corresponding to the "center of mass" of the spectrum.

Computed on the band-passed data; frequency-input domain.

$$
\text { Spectral centroid }=\frac{\sum_{k=0}^{N / 2} \frac{k}{N T}\left|M_{k}\right|^{2}}{\sum_{k=0}^{N / 2}\left|M_{k}\right|^{2}}
$$

3) Spectral Peak: The frequency corresponding to the peak of the estimated power spectral density. Computed on raw data; frequency-input domain.

$$
\text { Spectral peak }=\frac{1}{N T} \underset{k}{\operatorname{argmax}}\left|M_{k}\right|^{2}, k \in\left\{0,1, \ldots \frac{N}{2}\right\}
$$


640 4) Line length after spectral equalization: Detections are first detrended and energy normalized 641 by dividing by their Euclidean lengths and by sample length. Computed on raw data after first642 order backward differencing; time-domain input.

$$
\text { Line length }=\frac{1}{L} \sum_{i=0}^{L-2}\left|x_{i-1}-x_{i}\right|
$$

643

644

645 5) Line length (bandpassed) per sample length: line length of the 100-500 Hz bandpassed HFO 646

$$
\text { Line length (bandpassed) per sample length }=\frac{1}{N} \sum_{i=2}^{N}\left|x_{i}-x_{i-1}\right|
$$

6) Zero-crossings per sample length: the number of times the sample crosses its mean divided by the total length of the sample, which is zero-meaned

$$
\text { Zero crossings per sample }=\frac{1}{N} \sum_{i=2}^{N} \operatorname{sign}\left(x_{i-1}\right) \neq \operatorname{sign}\left(x_{i}\right)
$$

7) Maximum amplitude range: the difference between a sample's maximum and minimum values

$$
\text { Max. amplitude range }=\max (x)-\min (x)
$$

8) Number of peaks per sample length: the total number of local maxima in a sample divided by the total length of the sample

$$
\text { No. peaks per sample }=\frac{1}{N} \sum_{i=2}^{N-1}\left(x_{i}>x_{i-1} \wedge x_{i}>x_{i+1}\right)
$$




\section{Appendix C: Classifier Cross Validation}

658 The k-NN classifier requires specification of the value for $k$, the number of nearest neighbors to

659 use. We determined the optimal $k$ by calculating the average performance of the classifier for a

660 range of $k$ values (from 1 to 20 ) over the ten partitions of training and validation data and

661 selecting the input parameter that corresponded to the best average performance. We similarly

662 obtained optimal parameters for the support vector machine (SVM) classifier, which requires

663 specification of a) a cost parameter, $C$, that controls the tradeoff between reducing training errors

664 and controlling the complexity of the model, and b) a parameter, $\gamma$, that controls the width of the

665 Gaussian kernel. The latter two classifiers were re-trained on the augmented training and

666 validation data using these optimal parameters. We did not optimize any parameters for the

667 logistic regression classifier.

668

669

670 
Akiyama T, McCoy B, Go CY, Ochi A, Elliott IM, Akiyama M, Donner EJ, Weiss SK, Snead OC, 3rd, Rutka JT, Drake JM, and Otsubo H. Focal resection of fast ripples on extraoperative intracranial EEG improves seizure outcome in pediatric epilepsy. Epilepsia 52: 1802-1811, 2011. Benar CG, Chauviere L, Bartolomei F, and Wendling F. Pitfalls of high-pass filtering for detecting epileptic oscillations: a technical note on "false" ripples. Clin Neurophysiol 121: 301$310,2010$. Litt B, and Worrell GA. Data mining neocortical high-frequency oscillations in epilepsy and controls. Brain 134: 2948-2959, 2011.

Blanco JA, Stead M, Krieger A, Viventi J, Marsh WR, Lee KH, Worrell GA, and Litt B. Unsupervised classification of high-frequency oscillations in human neocortical epilepsy and control patients. Journal of neurophysiology 104: 2900-2912, 2010.

Bragin A, Benassi SK, Kheiri F, and Engel J, Jr. Further evidence that pathologic highfrequency oscillations are bursts of population spikes derived from recordings of identified cells in dentate gyrus. Epilepsia 52: 45-52, 2011.

Bragin A, Wilson CL, Staba RJ, Reddick M, Fried I, and Engel J, Jr. Interictal highfrequency oscillations $(80-500 \mathrm{~Hz})$ in the human epileptic brain: entorhinal cortex. Annals of neurology 52: 407-415, 2002a.

Bragin A, Mody I, Wilson CL, and Engel J, Jr. Local generation of fast ripples in epileptic brain. J Neurosci 22: 2012-2021, 2002b.

Bragin A, Wilson CL, Almajano J, Mody I, and Engel J, Jr. High-frequency oscillations after status epilepticus: epileptogenesis and seizure genesis. Epilepsia 45: 1017-1023, 2004.

Bragin A, Wilson CL, and Engel J, Jr. Voltage depth profiles of high-frequency oscillations after kainic acid-induced status epilepticus. Epilepsia 48 Suppl 5: 35-40, 2007.

Buzsaki G. Memory consolidation during sleep: a neurophysiological perspective. J Sleep Res 7 Suppl 1: 17-23, 1998.

700 Demont-Guignard S, Benquet P, Gerber U, Biraben A, Martin B, and Wendling F. Distinct hyperexcitability mechanisms underlie fast ripples and epileptic spikes. Annals of neurology 71: 342-352, 2012.

Dzhala VI, and Staley KJ. Mechanisms of fast ripples in the hippocampus. J Neurosci 24: 8896-8906, 2004.

Engel J, Jr., Bragin A, Staba R, and Mody I. High-frequency oscillations: what is normal and what is not? Epilepsia 50: 598-604, 2009.

Foffani G, Uzcategui YG, Gal B, and Menendez de la Prida L. Reduced spike-timing reliability correlates with the emergence of fast ripples in the rat epileptic hippocampus. Neuron 55: 930-941, 2007.

Gardner AB, Worrell GA, Marsh E, Dlugos D, and Litt B. Human and automated detection of high-frequency oscillations in clinical intracranial EEG recordings. Clin Neurophysiol 118: 1134-1143, 2007. Grenier F, Timofeev I, and Steriade M. Focal synchronization of ripples $(80-200 \mathrm{~Hz})$ in neocortex and their neuronal correlates. Journal of neurophysiology 86: 1884-1898, 2001. Ibarz JM, Foffani G, Cid E, Inostroza M, and Menendez de la Prida L. Emergent dynamics 
of fast ripples in the epileptic hippocampus. $J$ Neurosci 30: 16249-16261, 2010.

Jacobs J, Zelmann R, Jirsch J, Chander R, Dubeau CE, and Gotman J. High frequency oscillations $(80-500 \mathrm{~Hz})$ in the preictal period in patients with focal seizures. Epilepsia 2009. Jacobs J, Zijlmans M, Zelmann R, Chatillon CE, Hall J, Olivier A, Dubeau F, and Gotman J. High-frequency electroencephalographic oscillations correlate with outcome of epilepsy surgery. Annals of neurology 67: 209-220, 2010.

Jefferys JG, de la Prida LM, Wendling F, Bragin A, Avoli M, Timofeev I, and Lopes da Silva FH. Mechanisms of physiological and epileptic HFO generation. Prog Neurobiol 2012. Khosravani H, Mehrotra N, Rigby M, Hader WJ, Pinnegar CR, Pillay N, Wiebe S, and Federico P. Spatial localization and time-dependant changes of electrographic high frequency oscillations in human temporal lobe epilepsy. Epilepsia 50: 605-616, 2009.

Khosravani H, Pinnegar CR, Mitchell JR, Bardakjian BL, Federico P, and Carlen PL. Increased high-frequency oscillations precede in vitro low-Mg seizures. Epilepsia 46: 11881197, 2005.

Menendez de la Prida L, and Trevelyan AJ. Cellular mechanisms of high frequency oscillations in epilepsy: on the diverse sources of pathological activities. Epilepsy Res 97: 308317, 2011.

Mormann F, Andrzejak RG, Elger CE, and Lehnertz K. Seizure prediction: the long and winding road. Brain 130: 314-333, 2007.

Roopun AK, Simonotto JD, Pierce ML, Jenkins A, Nicholson C, Schofield IS, Whittaker RG, Kaiser M, Whittington MA, Traub RD, and Cunningham MO. A nonsynaptic mechanism underlying interictal discharges in human epileptic neocortex. Proc Natl Acad Sci $U$ $S$ A 107: 338-343, 2010.

Siapas AG, and Wilson MA. Coordinated interactions between hippocampal ripples and cortical spindles during slow-wave sleep. Neuron 21: 1123-1128, 1998.

Snyder DE, Echauz J, Grimes DB, and Litt B. The statistics of a practical seizure warning system. J Neural Eng 5: 392-401, 2008.

Staba RJ, Wilson CL, Bragin A, Fried I, and Engel J, Jr. Quantitative analysis of highfrequency oscillations $(80-500 \mathrm{~Hz})$ recorded in human epileptic hippocampus and entorhinal cortex. Journal of neurophysiology 88: 1743-1752, 2002.

Stacey WC, Krieger A, and Litt B. Network recruitment to coherent oscillations in a hippocampal computer model. Journal of neurophysiology 105: 1464-1481, 2011.

Stacey WC and Litt B. Technology insight: designing devices for seizure control. Nature clinical practice 4: 190-201, 2008.

Stacey WC, Lazarewicz MT, and Litt B. Synaptic Noise and Physiological Coupling Generate High Frequency Oscillations in a Hippocampal Computational Model. Journal of neurophysiology 102: 2342-2357, 2009.

Traub RD. Fast Oscillations and Epilepsy. Epilepsy Curr 3: 77-79, 2003. Urrestarazu E, Chander R, Dubeau F, and Gotman J. Interictal high-frequency oscillations (100-500 Hz) in the intracerebral EEG of epileptic patients. Brain 130: 2354-2366, 2007. Van Gompel JJ, Stead SM, Giannini C, Meyer FB, Marsh WR, Fountain T, So E, CohenGadol A, Lee KH, and Worrell GA. Phase I trial: safety and feasibility of intracranial electroencephalography using hybrid subdural electrodes containing macro- and microelectrode arrays. Neurosurg Focus 25: E23, 2008.

Wendling F, Bartolomei F, Mina F, Huneau C, and Benquet P. Interictal spikes, fast ripples and seizures in partial epilepsies - combining multi-level computational models with 
762 experimental data. Eur J Neurosci 36: 2164-2177, 2012.

763 Worrell GA, Gardner AB, Stead SM, Hu S, Goerss S, Cascino GJ, Meyer FB, Marsh R,

764 and Litt B. High-frequency oscillations in human temporal lobe: simultaneous microwire and

765 clinical macroelectrode recordings. Brain 131: 928-937, 2008.

766 Ylinen A, Bragin A, Nadasdy Z, Jando G, Szabo I, Sik A, and Buzsaki G. Sharp wave-

767 associated high-frequency oscillation $(200 \mathrm{~Hz})$ in the intact hippocampus: network and

768 intracellular mechanisms. J Neurosci 15: 30-46, 1995.

769 Zijlmans M, Jacobs J, Kahn YU, Zelmann R, Dubeau F, and Gotman J. Ictal and interictal

770 high frequency oscillations in patients with focal epilepsy. Clin Neurophysiol 122: 664-671,

7712011.

772 Zijlmans M, Jacobs J, Zelmann R, Dubeau F, and Gotman J. High frequency oscillations

773 and seizure frequency in patients with focal epilepsy. Epilepsy Res 85: 287-292, 2009.

774

775 
778 Ms. Pearce was supported by the Rachleff Scholars Program in Engineering at the University of 779 Pennsylvania. University of Pennsylvania students and investigators were supported by US 780 National Institutes of Health: National Institute of Neurological Disorders and Stroke (NINDS) 781 2R01 NS041811-06 and 1 U24 NS063930-02, Citizens United for Research in Epilepsy, and the 782 Dr. Michel and Mrs. Anna Mirowski Discovery Fund for Epilepsy Research. Dr. Stacey’s work 783 is supported by NINDS 1K08NS069783-02. Special thanks to the authors of (Blanco et al. 784 2011) for sharing their EEG data and HFO markings on the IEEG-Portal (www.ieeg.org). 785 786 787 


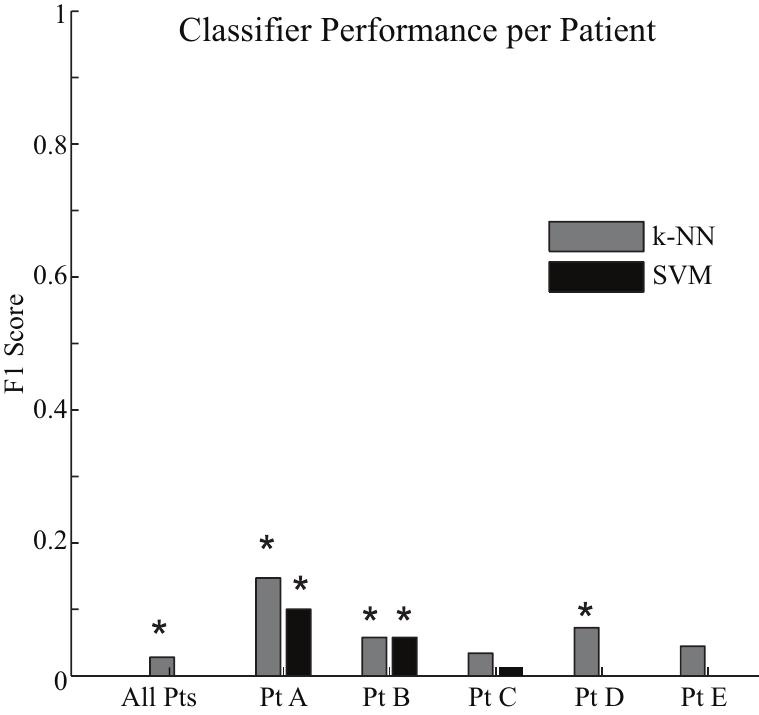




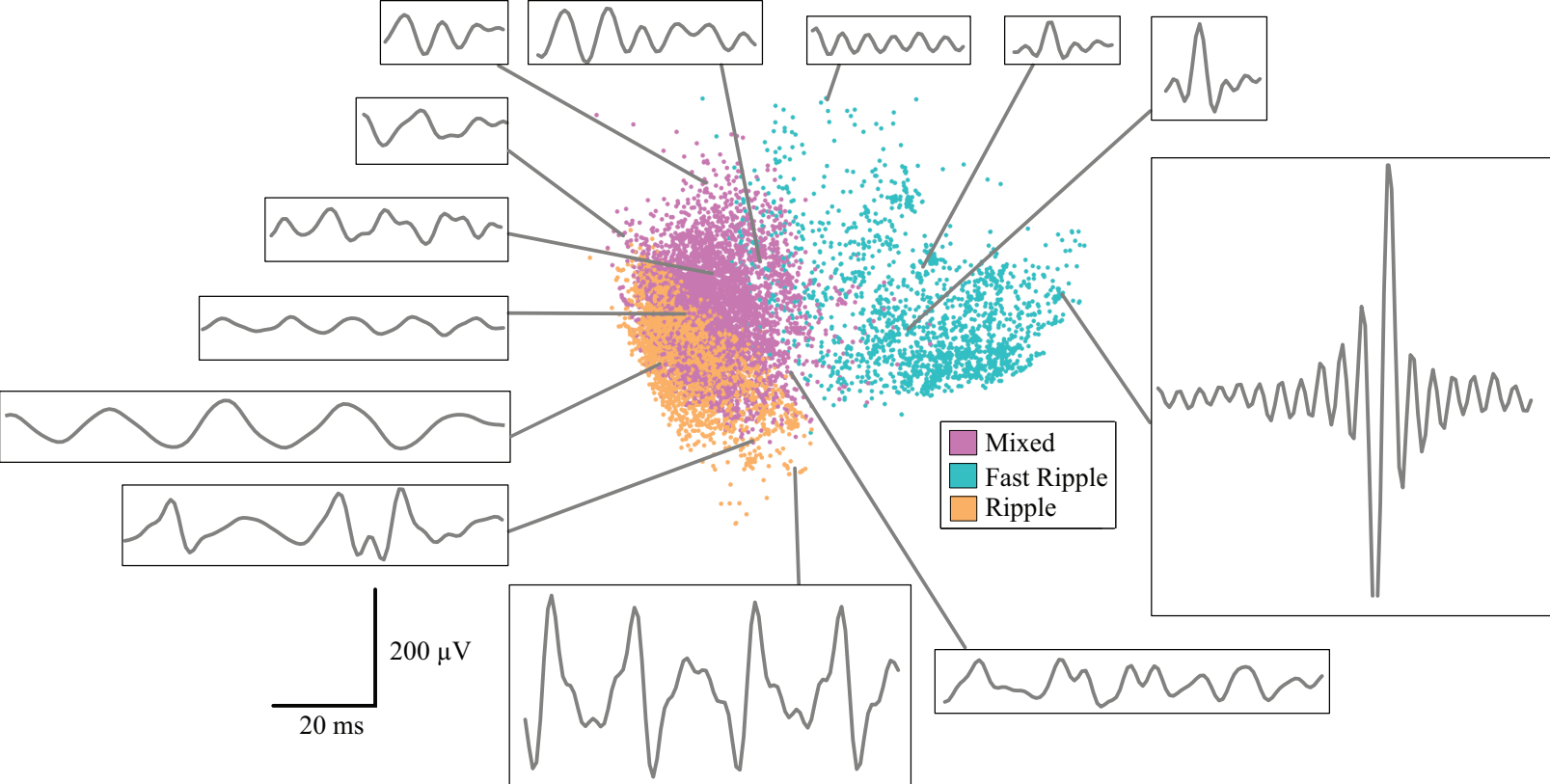




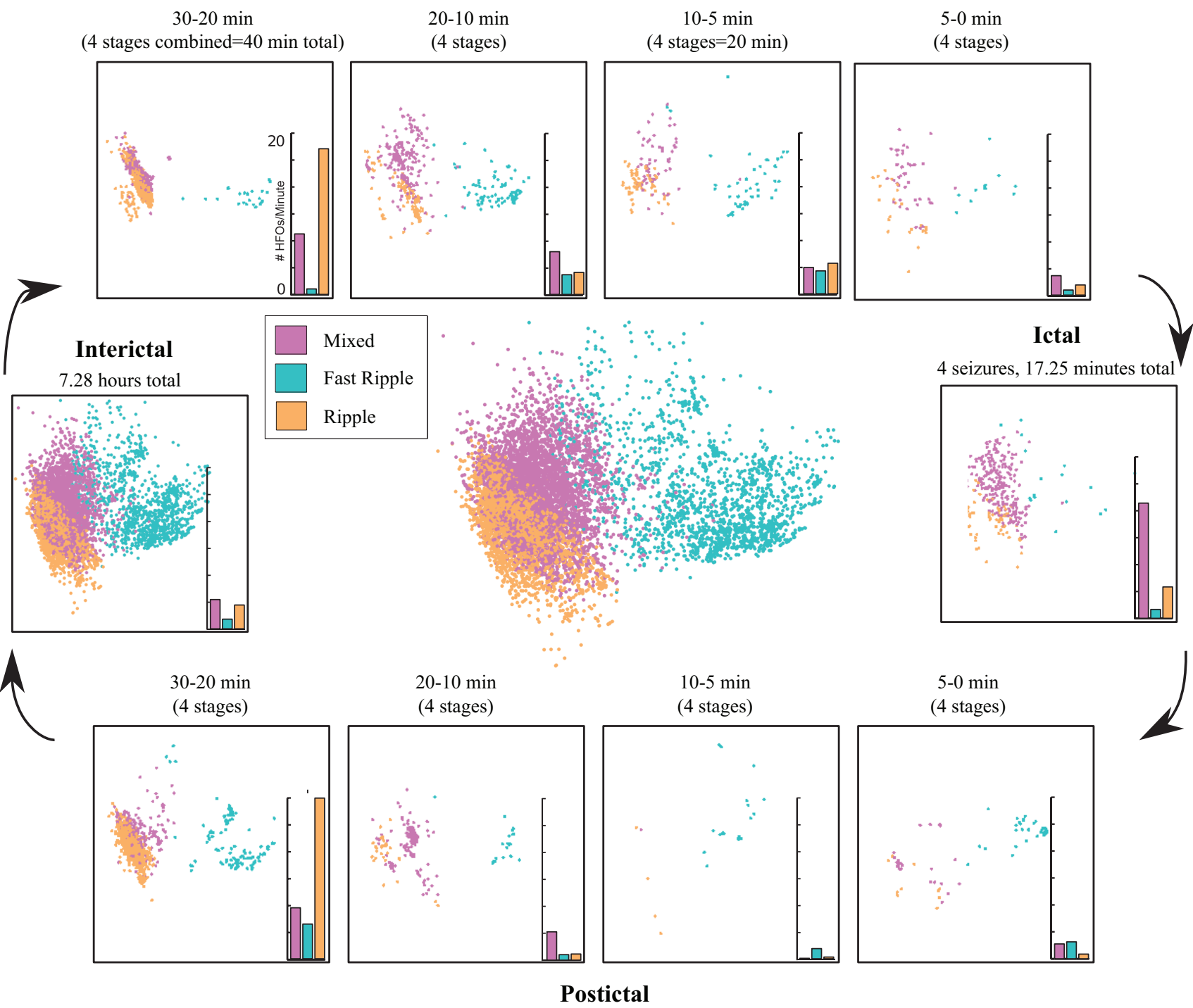




\section{Time to next seizure}

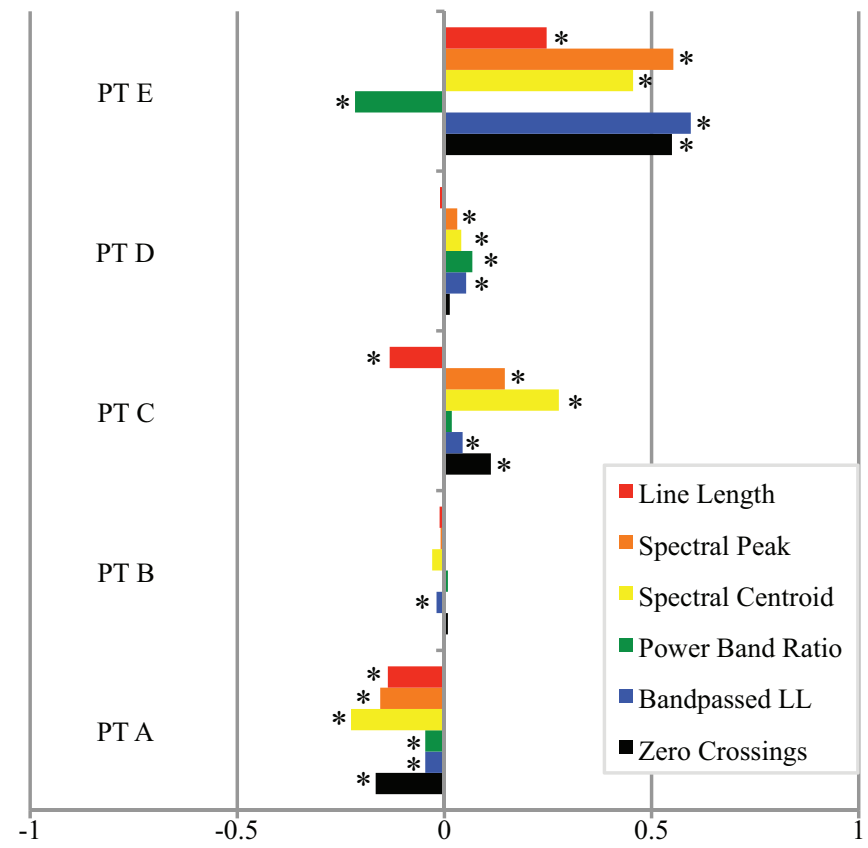

B

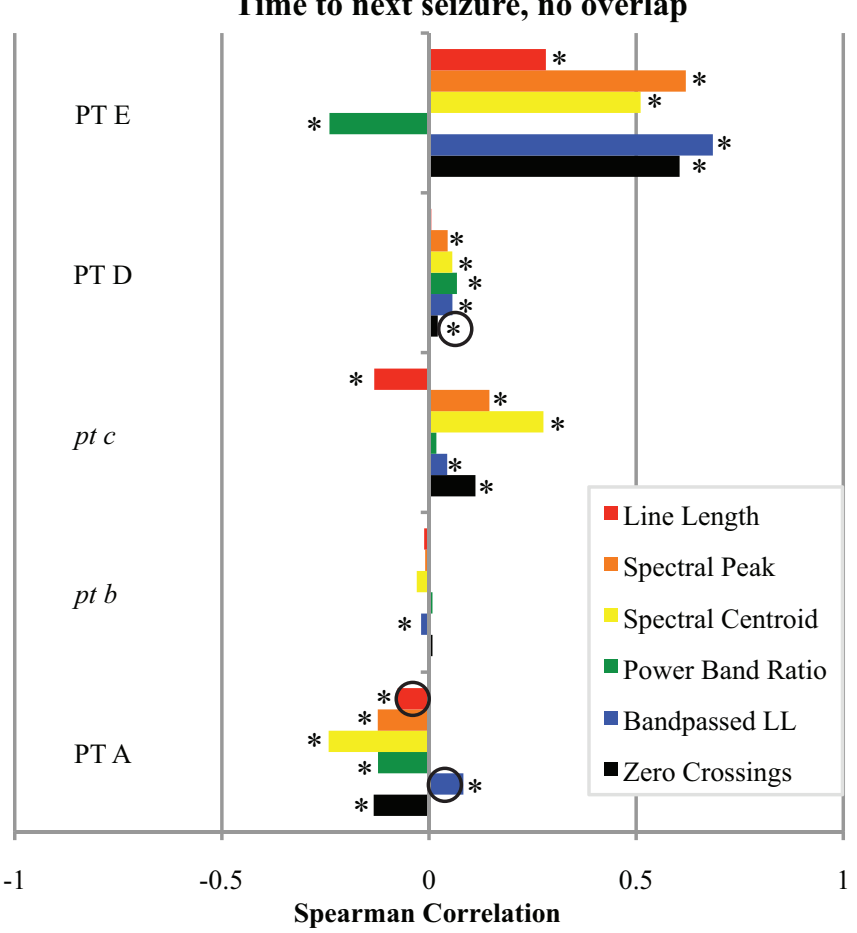

Time from previous seizure

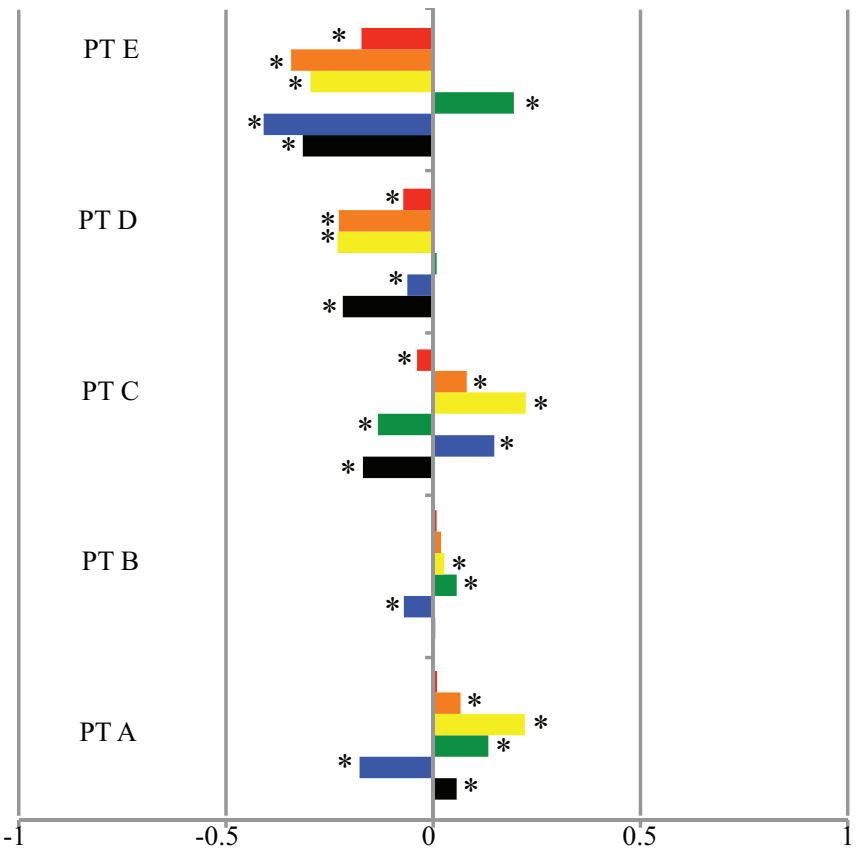

Time from previous seizure, no overlap

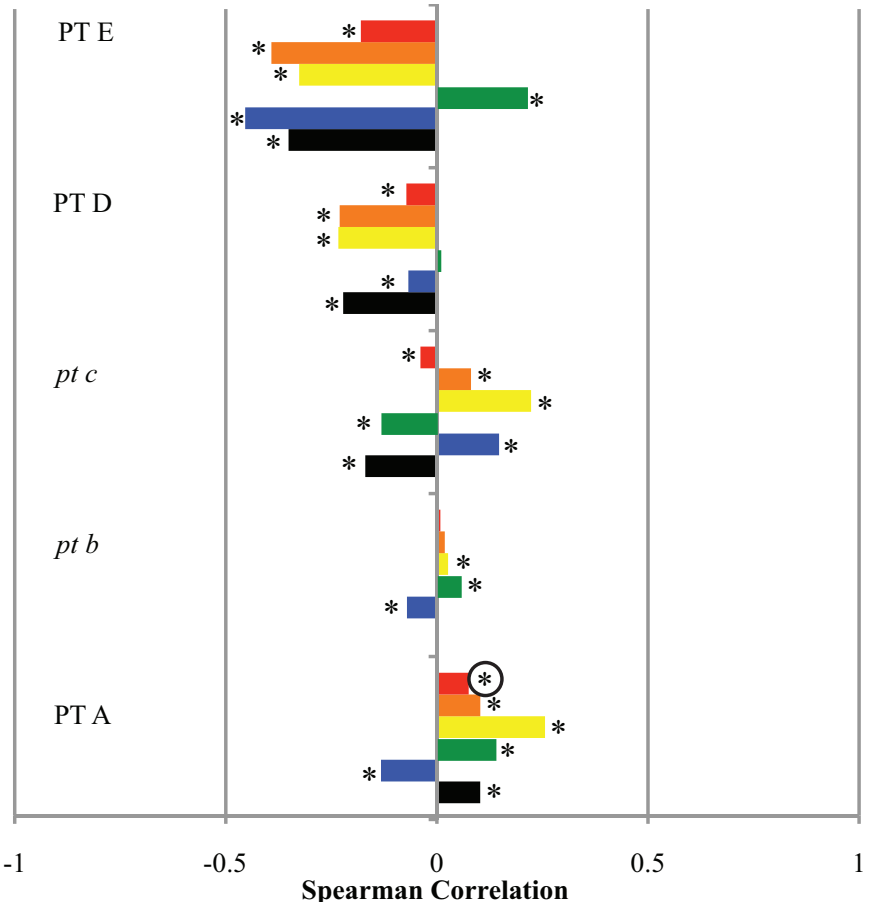




\section{Patient A (9 seizures)}

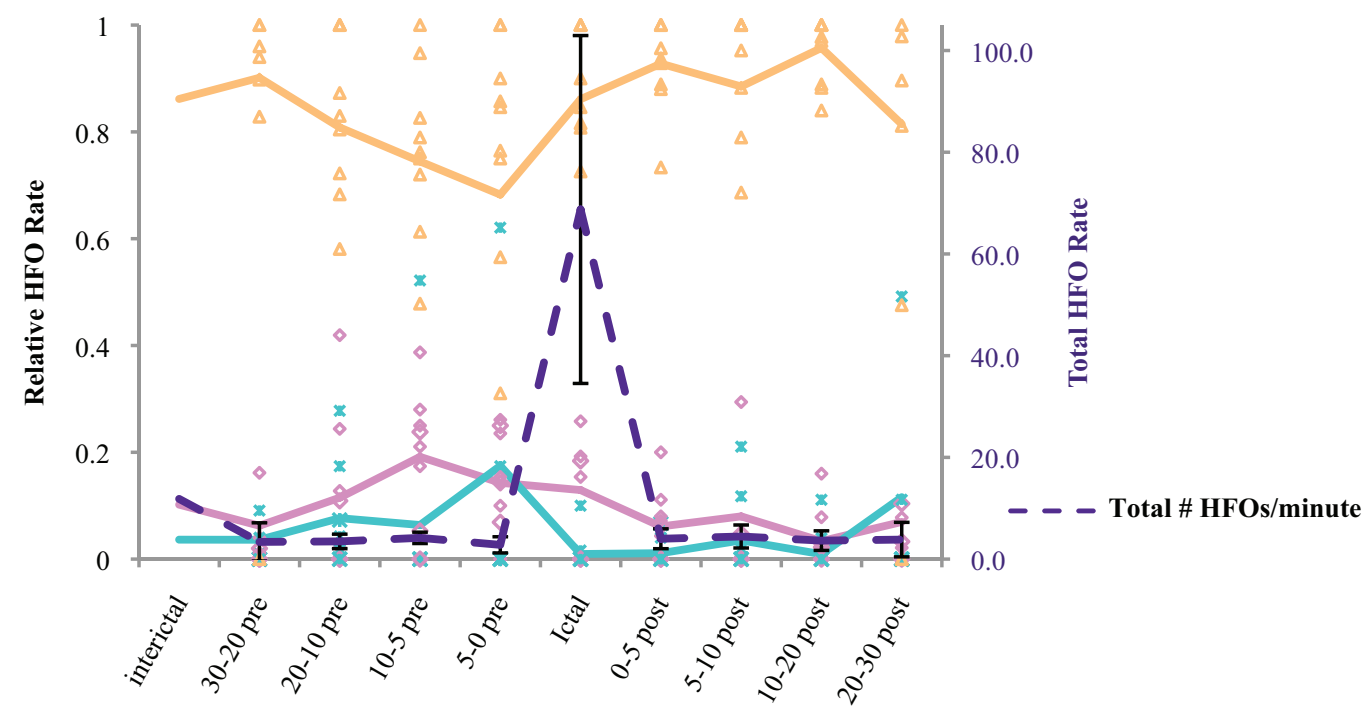

Patient B (3)

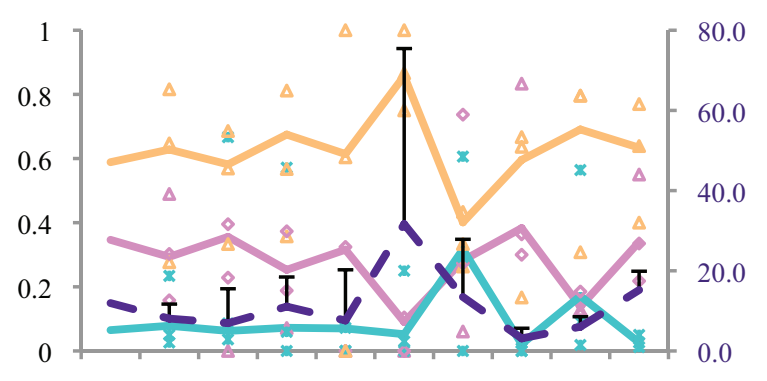

हैं

Patient D (7)

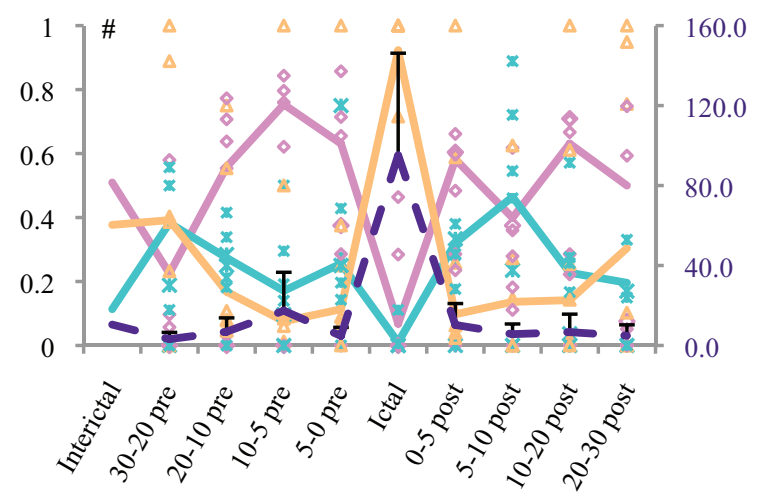

Patient C (4)

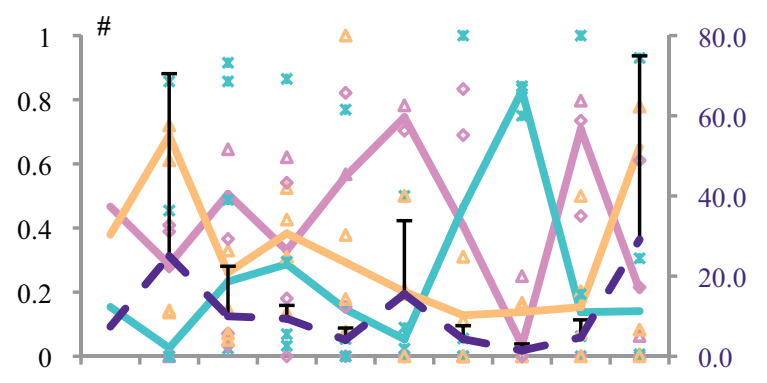

हैं

Patient E (2)

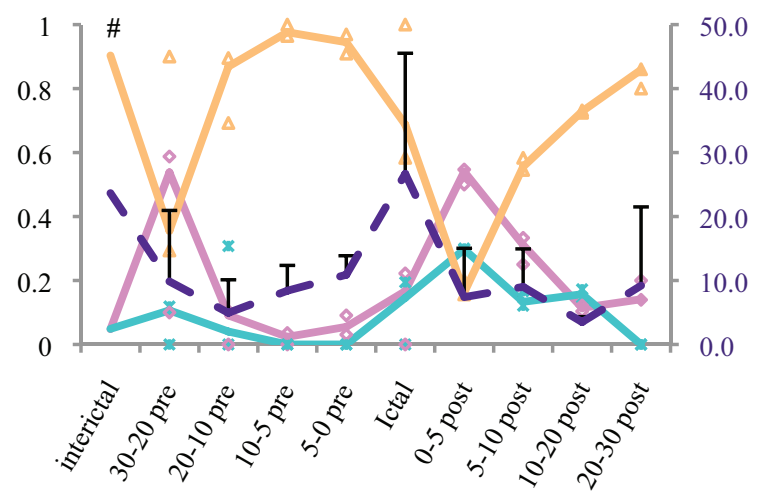


Table 1: Patient summary

\begin{tabular}{|c|c|c|c|c|c|}
\hline Subject & Age & Sex & Pathology & Electrodes & Electrode Placement \\
\hline $\begin{array}{c}\mathrm{A} \\
(\mathrm{SZ} 01)\end{array}$ & 35 & $\mathrm{~F}$ & $\begin{array}{l}\text { Frontal neocortical } \\
\text { oligodendroglioma }\end{array}$ & $\begin{array}{l}\text { Standard: } 6 \times 6 \\
\text { Micro: } 1 \text { x } 8\end{array}$ & Right: frontal, motor cortex \\
\hline $\begin{array}{c}\mathrm{B} \\
(\mathrm{SZ02})\end{array}$ & 24 & M & $\begin{array}{l}\text { Frontal cortical } \\
\text { dysplasia }\end{array}$ & $\begin{array}{l}\text { Standard: } 6 \times 6 \\
\text { Micro: } 4 \times 6\end{array}$ & Left: frontal, frontoparietal \\
\hline $\begin{array}{c}\mathrm{C} \\
(\mathrm{SZ04})\end{array}$ & 39 & $\mathrm{~F}$ & $\begin{array}{c}\text { Temporal } \\
\text { neocortical gliosis }\end{array}$ & $\begin{array}{l}\text { Standard: } 6 \times 6,1 \times 8, \\
1 \times 8,1 \times 4,1 \times 4,1 \times 4 \\
\text { Micro: } 1 \times 4\end{array}$ & $\begin{array}{l}\text { Left: temporal, inferior frontal, superior } \\
\text { frontal, anterior temporal, posterior } \\
\text { temporal, anterior, posterior }\end{array}$ \\
\hline $\begin{array}{c}\mathrm{D} \\
(\mathrm{SZ07})\end{array}$ & 42 & $\mathrm{~F}$ & $\begin{array}{l}\text { Frontal cortical } \\
\text { dysplasia }\end{array}$ & $\begin{array}{l}\text { Standard: } 6 \times 8,3 \times 8 \\
1 \times 4 \\
\text { Micro: } 1 \times 4,1 \times 8\end{array}$ & $\begin{array}{l}\text { Right: frontal, temporal, anterior } \\
\text { temporal, inferior temporal, posterior } \\
\text { temporal }\end{array}$ \\
\hline $\begin{array}{c}\mathrm{E} \\
(\mathrm{SZ08})\end{array}$ & 38 & $\mathrm{~F}$ & $\begin{array}{c}\text { Temporal } \\
\text { neocortical gliosis }\end{array}$ & $\begin{array}{l}\text { Standard: 6x6 } \\
\text { Standard depth: } \\
1 \mathrm{x} 4 \\
\text { Micro depth: } 1 \times 4, \\
1 \mathrm{x} 4\end{array}$ & $\begin{array}{l}\text { Left: temporal, anterior temporal, } \\
\text { middle temporal, posterior temporal }\end{array}$ \\
\hline
\end{tabular}

Summary of patient clinical characteristics and electrode placement. Parentheses: labels used in prior study, see Table 1 in (Blanco et al. 2011) for further details. 
Table 2: HFO and seizure counts

\begin{tabular}{|c|r|r|r|r|r|r|}
\hline Patient & $\begin{array}{c}\text { Total } \\
\text { seizures }\end{array}$ & $\begin{array}{c}\text { Total } \\
\text { HFOs }\end{array}$ & $\begin{array}{c}\text { Inter- } \\
\text { ictal }\end{array}$ & Preictal & Ictal & $\begin{array}{r}\text { Post- } \\
\text { ictal }\end{array}$ \\
\hline A & 9 & 2552 & 1487 & 314 & 371 & 380 \\
\hline B & 3 & 9905 & 9237 & 280 & 139 & 249 \\
\hline C & 4 & 12417 & 11585 & 329 & 317 & 186 \\
\hline D & 7 & 53015 & 49519 & 1787 & 433 & 1276 \\
\hline E & 2 & 8262 & 7210 & 493 & 46 & 513 \\
\hline All & 25 & 86151 & 79038 & 3206 & 1306 & 2604 \\
\hline
\end{tabular}

Counts of HFOs in each time epoch for dispersion analysis. Preictal and postictal epochs were defined as 10 minutes before and after seizures, respectively. 
Table 3: Dispersion p-values from 2 features

\begin{tabular}{|l|r|r|r|r|r|r|}
\cline { 2 - 7 } \multicolumn{1}{c|}{} & \multicolumn{7}{|c|}{ Feature 3 (Line length) } \\
\cline { 2 - 7 } \multicolumn{1}{c|}{} & Pt A & Pt B & Pt C & Pt D & Pt E & All \\
\hline Interictal & 0.0018 & $*$ & $*$ & $*$ & 0.0011 & $*$ \\
\hline Preictal & $\mathbf{0 . 3 7 1 3}$ & $\mathbf{0 . 0 6 1 5}$ & $*$ & $*$ & 0.0002 & $\mathbf{0 . 2 2 4 2}$ \\
\hline Ictal & 0.0426 & 0.0007 & $*$ & $\mathbf{0 . 3 1 4 8}$ & 0.0155 & $*$ \\
\hline Postictal & $\mathbf{0 . 0 9 8 3}$ & $*$ & $*$ & 0.0038 & $*$ & $*$ \\
\hline
\end{tabular}

\begin{tabular}{|l|r|r|r|r|r|r|}
\cline { 2 - 7 } \multicolumn{1}{c|}{} & \multicolumn{6}{c|}{ Feature 4 (Power Band Ratio) } \\
\cline { 2 - 7 } \multicolumn{1}{c|}{} & Pt A & Pt B & Pt C & Pt D & Pt E & All \\
\hline Interictal & $*$ & $\mathbf{0 . 2 2 8 5}$ & $*$ & $*$ & $*$ & $\mathbf{0 . 1 3 9 8}$ \\
\hline Preictal & 0.0442 & $\mathbf{0 . 4 9 1 6}$ & $*$ & $*$ & $\mathbf{0 . 4 2 0 8}$ & $*$ \\
\hline Ictal & $*$ & 0.0001 & $*$ & $*$ & $*$ & $*$ \\
\hline Postictal & $*$ & $\mathbf{0 . 1 0 5 9}$ & $*$ & $*$ & $*$ & $\mathbf{0 . 3 2 8 5}$ \\
\hline
\end{tabular}

Table 3: P-values from the epoch dispersion analysis. Values were calculated by dividing the number of permutation test trials with results more extreme than the true values by the total number of permutation test trials $(10,000)$. Bold text indicates values that were not statistically significant. The combination of all (six) features was significant in all patients. Each patient had different trends with the individual features. Asterisks: highly significant $(p<1 \mathrm{e}-6)$ 
Table 4: P-values of temporal rank

\begin{tabular}{|l|l|l|l|}
\cline { 2 - 4 } \multicolumn{1}{c|}{} & $\begin{array}{l}\text { Time to } \\
\text { Next }\end{array}$ & $\begin{array}{l}\text { Time From } \\
\text { Previous }\end{array}$ & $\begin{array}{l}\text { Closest } \\
\text { cluster (next) }\end{array}$ \\
\hline Pt A & $<.0001^{*}$ & $<.0001^{*}$ & FR, M \\
\hline Pt B & $0.013^{*}$ & $<.0001^{*}$ & M, R \\
\hline Pt C & $<.0001^{*}$ & $<.0001^{*}$ & FR, R \\
\hline Pt D & $<.0001^{*}$ & $<.0001^{*}$ & FR, R \\
\hline Pt E & $<.0001^{*}$ & $<.0001^{*}$ & R \\
\hline
\end{tabular}

Table 4: P-values from the second Kruskal-Wallis Test, used to assess whether an HFO near a seizure was more likely to be of a specific cluster. Third column indicates which clusters were closest to the next seizure (R: ripple, M: mixed, FR: fast ripple ). Asterisks: statistical significance. 
